\title{
Rosiglitazone promotes development of a novel adipocyte population from bone marrow-derived circulating progenitor cells
}

\author{
Joseph T. Crossno Jr., 1,2,3 Susan M. Majka,2,4 Todd Grazia,3,5 \\ Ronald G. Gill, ${ }^{5}$ and Dwight J. Klemm¹,2,3

\begin{abstract}
${ }^{1}$ Research Section, Veterans Affairs Medical Center, Denver, Colorado, USA. ${ }^{2}$ Cardiovascular Pulmonary Research Laboratory,
${ }^{3}$ Division of Pulmonary Sciences and Critical Care Medicine, Department of Medicine, ${ }^{4}$ Division of Cardiology, and

${ }^{5}$ Barbara Davis Center for Childhood Diabetes, University of Colorado Health Sciences Center, Denver, Colorado, USA.
\end{abstract}

\begin{abstract}
Obesity and weight gain are characterized by increased adipose tissue mass due to an increase in the size of individual adipocytes and the generation of new adipocytes. New adipocytes are believed to arise from resident adipose tissue preadipocytes and mesenchymal progenitor cells. However, it is possible that progenitor cells from other tissues, in particular BM, could also contribute to development of new adipocytes in adipose tissue. We tested this hypothesis by transplanting whole BM cells from GFP-expressing transgenic mice into wild-type C57BL/6 mice and subjecting them to a high-fat diet or treatment with the thiazolidinedione (TZD) rosiglitazone (ROSI) for several weeks. Histological examination of adipose tissue or FACS of adipocytes revealed the presence of $\mathrm{GFP}^{+}$multilocular (ML) adipocytes, whose number was significantly increased by ROSI treatment or high-fat feeding. These ML adipocytes expressed adiponectin, perilipin, fatty acid-binding protein (FABP), leptin, $\mathrm{C} / \mathrm{EBP} \alpha$, and PPAR $\gamma$ but not uncoupling protein-1 (UCP-1), the CD45 hematopoietic lineage marker, or the CDllb monocyte marker. They also exhibited increased mitochondrial content. Appearance of GFP ${ }^{+} \mathrm{ML}$ adipocytes was contemporaneous with an increase in circulating levels of mesenchymal and hematopoietic progenitor cells in ROSI-treated animals. We conclude that TZDs and high-fat feeding promote the trafficking of BM-derived circulating progenitor cells to adipose tissue and their differentiation into ML adipocytes.
\end{abstract}

\section{Introduction}

Adipose tissue is a primary site for energy storage and also acts as an endocrine organ that regulates energy homeostasis via the secretion of adipokines such as adiponectin, leptin, and resistin (1). The origin and development of adipocytes and adipose tissue is not completely understood, but these processes are crucial to our knowledge of normal adipose tissue function and the adverse effects of excessive adiposity associated with weight gain and obesity.

Adipose tissue development begins during gestation in higher mammals $(2,3)$ and shortly after birth in rodents $(4)$. Dense regions of mesenchymal cells associated with vascular structures form at sites where adipose tissue ultimately develops. Within these regions, multipotent mesenchymal stem cells transform into unipotential adipoblasts that eventually become lineagecommitted preadipocytes. With appropriate stimulation, the preadipocytes undergo adipogenic conversion to mature, lipid-filled, insulin-sensitive adipocytes.

After birth and throughout life, adipose tissue can expand in response to elevated dietary energy intake via hypertrophy of existing fat cells and through the generation of new adipocytes (hyperplasia). Adipocyte hypertrophy is largely due to the accumulation of additional triglyceride from dietary sources $(5,6)$. Hyperplastic growth is generally attributed to the differentiation of resident

Nonstandard abbreviations used: APC, allophycocyanin; $\beta 3-\mathrm{AR}, \beta 3$-adrenergic

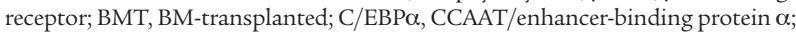
FABP, fatty acid-binding protein; ML, multilocular; MLWA, ML white adipocyte; PI, propidium iodide; ROSI, rosiglitazone; TZD, thiazolidinedione.

Conflict of interest: The authors have declared that no conflict of interest exists. Citation for this article: J. Clin. Invest. 116:3220-3228 (2006). doi:10.1172/JCI28510. preadipocytes and mesenchymal progenitor cells to form new adipocytes $(5,6)$. Adipose tissue mass also increases in response to treatment with thiazolidinediones (TZDs), which are used clinically as insulin-sensitizing, antidiabetic agents $(7,8)$. These agents stimulate the appearance of new adipocytes from resident preadipocytes and progenitor cells via their activation of the nuclear hormone receptor PPAR $\gamma(9,10)$.

Studies on adipocyte hyperplasia have focused almost entirely on resident preadipocytes and progenitor cells as the source of new adipocytes. However, there is ample reason to believe that a nonresident source of cells could also serve as a source of new adipocytes. Mesenchymal stem cells and multipotent progenitor cells can be isolated from many tissues and induced to differentiate into adipocytes in vitro (11-13). At the same time, weight gain, obesity, and treatment with TZDs are associated with changes in the circulating levels of cytokines and chemotactic factors (e.g., CCR2, CXCL10, and RANTES) that can regulate progenitor cell mobilization, trafficking, and recruitment $(14,15)$. Recent reports also indicate that TZDs promote the mobilization, homing, differentiation, and proliferation of BM-derived circulatory cells such as monocytes (14), endothelial progenitor cells (16), and platelets (17) to various tissues and organs, although trafficking to adipose tissue has not been examined. Thus, while there is reason to believe that nonresident progenitor cells contribute to the adipocyte population of adipose tissue, there is no direct evidence that this phenomenon occurs.

In this study, we set out to determine whether rosiglitazone (ROSI) or high-fat feeding could promote the mobilization of BMderived circulating progenitor cells to adipose tissue and promote their differentiation to adipocytes. We employed a model in which $\mathrm{GFP}^{+} \mathrm{BM}$-transplanted mice (BMT mice) were treated with ROSI 
Table 1

Effect of ROSI on cell-surface marker expression on PBMCs and omental and dorsal intrascapular fat stromal/vascular cells

\begin{tabular}{|c|c|c|c|c|c|c|}
\hline \multirow[b]{2}{*}{ Marker } & \multicolumn{2}{|c|}{ PBMCs } & \multicolumn{2}{|c|}{ Omental fat } & \multicolumn{2}{|c|}{ Dorsal fat } \\
\hline & Fold $\pm S D^{A}$ & $P$ & Fold $\pm S D^{A}$ & $P$ & Fold $\pm S D^{A}$ & $P$ \\
\hline CD45+Sca-1+ & $1.04 \pm 0.15^{B}$ & 0.25 & $1.03 \pm 0.08$ & 0.23 & $1.00 \pm 0.13$ & 0.49 \\
\hline CD $45^{+}$c-kit ${ }^{+}$ & $3.56 \pm 3.73^{B}$ & 0.12 & $1.02 \pm 0.08$ & 0.40 & $0.99 \pm 0.25$ & 0.44 \\
\hline $\mathrm{CD} 45^{+} \mathrm{CD} 34^{+}$ & $1.54 \pm 0.44$ & 0.21 & $0.94 \pm 0.30$ & 0.35 & $0.80 \pm 0.20$ & 0.36 \\
\hline CD45+CD11b+ & $0.92 \pm 0.11$ & 0.37 & $0.91 \pm 0.55$ & 0.47 & $0.67 \pm 0.12$ & 0.08 \\
\hline $\mathrm{CD} 45^{+} \mathrm{CD} 14^{+}$ & $2.01 \pm 1.19^{B}$ & 0.08 & $1.03 \pm 0.15$ & 0.43 & $0.92 \pm 0.18$ & 0.31 \\
\hline $\mathrm{CD} 45^{+} \mathrm{Gr}-1^{+}$ & $1.28 \pm 0.12^{\mathrm{B}}$ & 0.003 & $1.02 \pm 0.11$ & 0.39 & $0.68 \pm 0.35$ & 0.21 \\
\hline CD45+Thy-1+ & $1.20 \pm 0.09$ & 0.13 & $0.93 \pm 0.12$ & 0.19 & $0.98 \pm 0.02$ & 0.25 \\
\hline $\mathrm{CD} 45^{+} \mathrm{B}^{2} 2 \mathrm{O}^{+}$ & $0.90 \pm 0.14$ & 0.30 & $0.84 \pm 0.12$ & 0.07 & $1.21 \pm 0.20$ & 0.19 \\
\hline CD45-Sca-1+ & $7.74 \pm 1.66^{\mathrm{B}}$ & 0.02 & $0.53 \pm 0.04$ & 0.02 & $1.27 \pm 0.32$ & 0.30 \\
\hline
\end{tabular}

PBMCs and stromal/vascular cells from omental and intrascapular fat depots were isolated, treated with ROSI for 3 weeks, labeled with the cell-surface marker antibodies indicated in the left column, and subjected to flow cytometric analysis as described in Methods. AFold change in GFP+ cells expressing the indicated cell-surface markers from ROSI-treated animals relative to levels measured in samples from untreated animals. ${ }^{\mathrm{B}} n=5$. All other measurements, $n=2$ pools of cells from 3 animals. expressing transgenic mice, driven by the ubiquitin $\mathrm{C}$ gene promoter (18). The $\mathrm{GFP}^{+} \mathrm{BM}$ cells were allowed to engraft for 8 weeks, after which time greater than $95 \%$ of circulating PBMCs exhibited GFP expression (Supplemental Figure 1; supplemental material available online with this article; doi:10.1172/JCI28510DS1). $\mathrm{GFP}^{+} \mathrm{BMT}$ chimera mice were then fed either a conventional rodent chow diet (6.5\% fat, $3.31 \mathrm{kcal} / \mathrm{g})$, a diet impregnated with the TZD ROSI $(15 \mathrm{mg} / \mathrm{kg} / \mathrm{d})$, or a high-fat diet $(20 \%$ fat, $4.3 \mathrm{kcal} / \mathrm{g}$ ) for up to 7 weeks.

Flow cytometric analysis of BMderived, circulating $\mathrm{GFP}^{+}$PBMCs isolated from the peripheral blood or collagenase-digested omental or intrascapular fat pads showed no change in any of the populations following 1 week of ROSI or high-fat or high-fat diet. We found that a 3- to 5-week treatment with ROSI increased circulating mesenchymal and hematopoietic progenitor cell levels. Histological examination of adipose tissue or FACS of isolated adipocytes revealed the presence of $\mathrm{GFP}^{+}$multilocular (ML) adipocytes, whose number was significantly increased by ROSI treatment and to a lesser extent by high-fat feeding. Nuclear ploidy analysis confirmed that $\mathrm{GFP}^{+} \mathrm{ML}$ adipocytes do not arise from cell fusion events. The $\mathrm{GFP}^{+} \mathrm{ML}$ adipocytes expressed several adipocyte markers, but not the brown adipocyte marker uncoupling protein-1 (UCP-1) or the macrophage/monocyte marker CD11b. These GFP ${ }^{+}$ $\mathrm{ML}$ adipocytes also displayed increased mitochondrial activity. We conclude that treatment with TZDs and high-fat feeding increase the mobilization of BM-derived progenitor cells to adipose tissue and their differentiation into a novel ML adipocyte population.

\section{Results}

ROSI promotes mobilization of BM-derived mesenchymal progenitor cells into the peripheral circulation. To determine whether high-fat feeding or TZDs promote mobilization of BM-derived circulating progenitor cells to adipose tissue depots, C57BL/6 mice were subjected to lethal gamma irradiation (12 Gy split dose) to deplete their endogenous BM populations. Irradiated mice were rescued by isograft transplantation of isolated whole BM cells from GFP-

\section{Figure 1}

ROSI increases circulating levels of BM-derived mesenchymal and hematopoietic progenitor cells. Flow cytometric analysis of PBMCs isolated from GFP+ BMT mice fed a control (Cntrl) or ROSI-impregnated (ROSI) diet for 3 (top and bottom rows) or 7 (middle row) weeks. Cells were stained with APC-conjugated anti-CD45 antibodies and either PE-labeled anti-Sca-1 (top and middle rows) or anti-c-kit (bottom row) antibodies and analyzed by flow cytometry as described in Methods. Gates were set and data corrected for results obtained with unstained cells or cells stained with APC- or PE-conjugated isotype-matched control antibodies. Representative scattergrams for each analysis are shown. Blue ovals indicate cells staining weakly for Sca-1 that were not detected in samples from control animals. The average percentage (from 3 independent experiments) of total GFP+ cells is indicated in the top left, top right, and bottom right quadrants. diet treatment (Supplemental Table 1). However, after 3 weeks of ROSI treatment, there was a 7.74-fold increase $(P=0.02)$ in $\mathrm{GFP}^{+}$ cells expressing a pattern of cell-surface markers characteristic of mesenchymal progenitor cells (CD45-Sca- $1^{+}$) (Table 1 and Figure 1 ) in the peripheral blood mononuclear layer. There was also a 3.5-fold
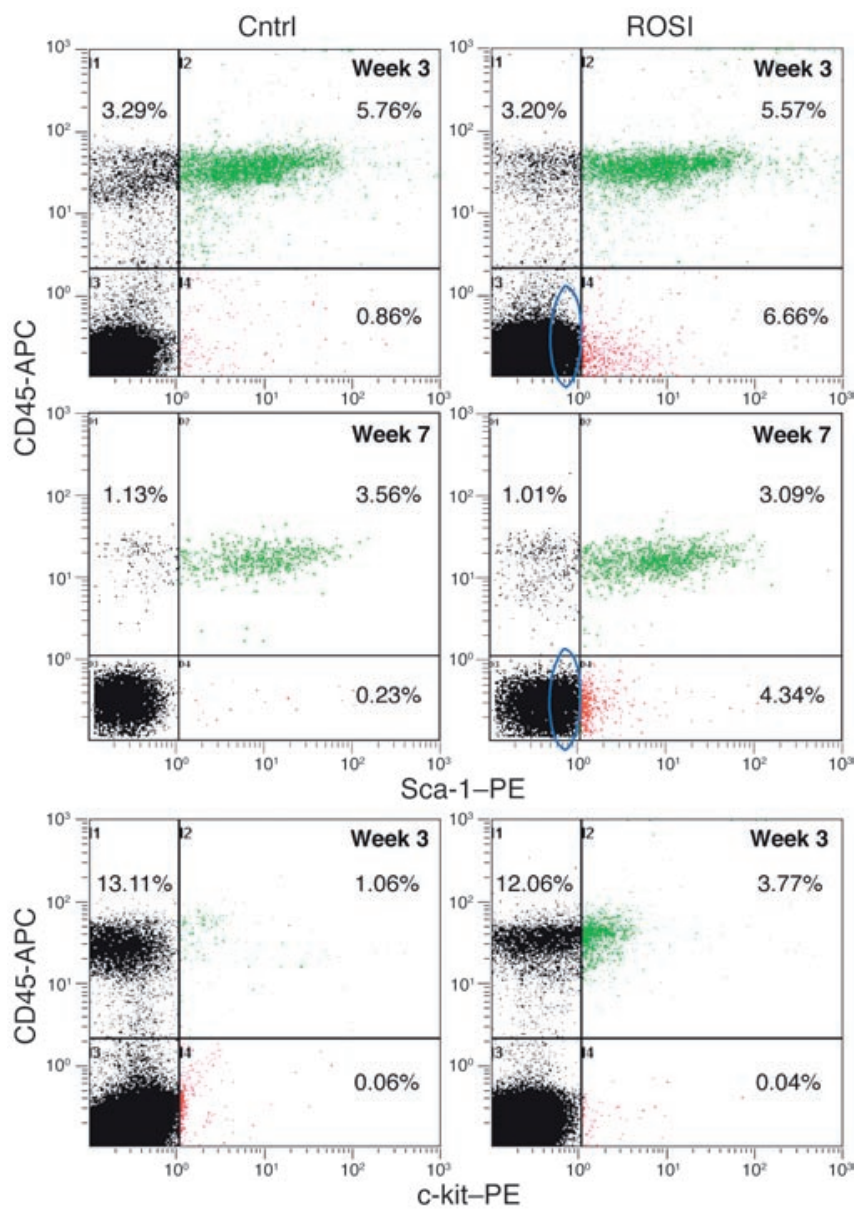
A
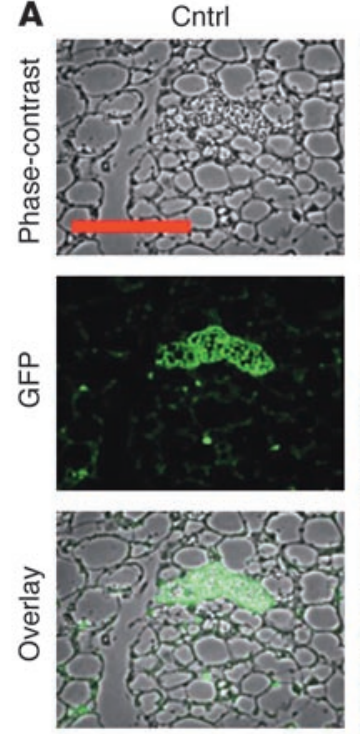

B

Phase-contrast

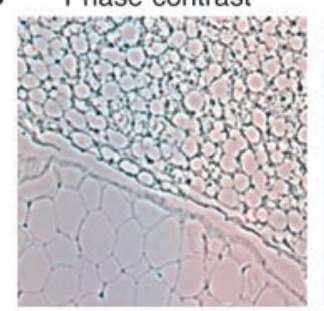

ROSI
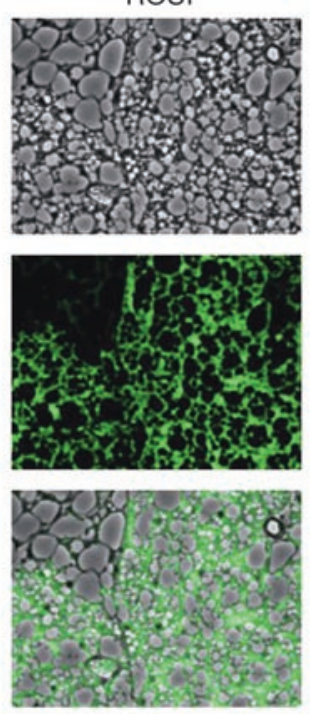

Iso match $\mathrm{Ab}$
High fat
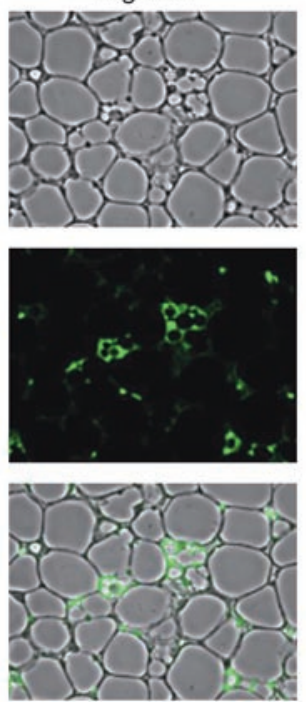

GFP Ab

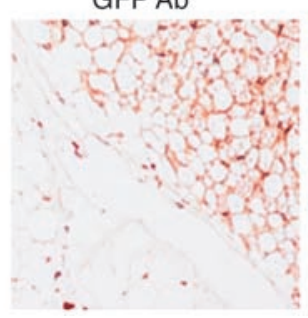

Brn fat ROSI
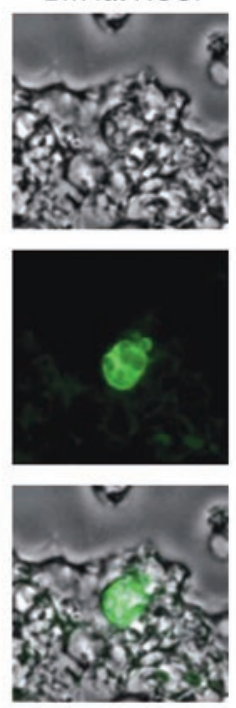

GFP fluorescence

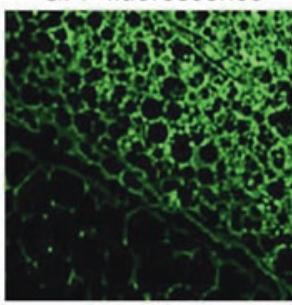

\section{Figure 2}

Appearance and distribution of GFP+ ML adipocytes in adipose tissue from untreated, ROSI-treated, and high-fat diet-fed mice. (A) Five-micrometer sections were prepared from paraffin-embedded omental (left 3 columns) and dorsal intrascapular (right column) adipose tissue from GFP+ BMT animals fed control, ROSI-impregnated, or high-fat diets for 3 weeks. Sections were deparaffinized, rehydrated, and mounted with aqueous mounting medium. Sections were examined by phase-contrast and fluorescence digital deconvolution microscopy. The GFP fluorescence signal was digitally overlayed on the corresponding phase-contrast image. Representative photomicrographs of both white fat (left 3 columns) and brown fat (Brn fat) are shown. Scale bar (red): $100 \mu \mathrm{m}$. (B) Serial sections of omental white fat from GFP+ BMT mice fed ROSI for 3 weeks were compared for GFP fluorescence and immunohistochemical staining for GFP (GFP Ab). Lack of staining with an isotype-matched negative control antibody (Iso match Ab) is also shown. increase in the number of peripheral blood circulating cells expressing surface markers characteristic of hematopoietic progenitor cells $\left(\mathrm{CD} 45^{+} \mathrm{c}-\mathrm{kit}{ }^{+}\right)$, although this change was not statistically significant $(P=0.12)$. Minor increases were also observed in circulating granulocytes $\left(\mathrm{Gr}-1^{+}\right)$and macrophages $\left(\mathrm{CD} 14^{+}\right)$. After 7 weeks of treatment, circulating levels of $\mathrm{GFP}^{+} \mathrm{CD} 45^{-} \mathrm{Sca}-1^{+}$cells were increased by almost 19-fold in ROSI-treated $\mathrm{GFP}^{+} \mathrm{BMT}$ chimera mice (Supplemental Table 1 and Figure 1). No other significant differences in peripheral blood circulating cell populations were noted between control and high-fat diet-fed animals.

Analysis of $\mathrm{GFP}^{+} \mathrm{BM}$-derived populations in the stromal/vascular fraction from collagenase-digested omental and intrascapular fat pads demonstrated a modest, but reproducible decrease in CD $45-\mathrm{Sca}-1^{+}$cells in omental fat with ROSI treatment at 3 (Table 1) and 7 (Supplemental Table 1) weeks. No significant differences were noted in other cell populations at any time point with any of the conditions. The fact that there were no significant differences in $\mathrm{GFP}^{+} \mathrm{BM}$-derived populations in adipose tissue with ROSI treatment while circulating levels of mesenchymal and hematopoietic progenitor cells were elevated by ROSI is likely due to the loss of progenitor cell-surface markers as these progenitor cells differentiated into adipocytes or other cell types.

$B M$-derived progenitor cells differentiate into ML adipocytes. Fluorescence digital deconvolution microscopy revealed $\mathrm{GFP}^{+}$adipocytes having multiple fat droplets (ML) in all treatment groups (Figure 2A). Endogenous GFP fluorescence or immunohistochemical staining for GFP indicated that these ML adipocytes arose from BM-derived progenitor cells (Figure 2B). These cells were more abundant in adipose tissue sections from ROSI-treated animals, often cluster- ing together in large regions. $\mathrm{ML} \mathrm{GFP}^{+}$adipocytes were also present at higher numbers in fat tissue from high-fat diet-fed animals than corresponding adipose tissue depots from control animals, but the levels were not as high as in samples from ROSI-treated animals. $\mathrm{GFP}^{+} \mathrm{ML}$ adipocytes were also present in intrascapular brown fat, but rather than forming clusters as observed in white fat, the $\mathrm{GFP}^{+}$adipocytes were observed as individual cells scattered throughout the brown fat.

To quantitate these differences, adipocytes were isolated from collagenase-digested omental and intrascapular fat and subjected to FACS analysis. A $100-\mu \mathrm{m}$ sample tip was selected for the sorting of adipocytes based on morphometric analysis of fixed adipose tissue sections and collagenase-digested adipocytes. These studies indicated that the average size of unilocular white adipocytes over all depots was approximately $40 \pm 15 \mu \mathrm{m}$ in control and ROSItreated animals and about $70 \pm 10 \mu \mathrm{m}$ in high-fat diet-fed animals. These sizes are somewhat smaller than typically reported. This is probably due to the fact that irradiated animals and/or animals maintained at Denver, Colorado, altitude (1,600 m) eat less, gain less weight, and exhibit decreased adiposity compared with unirradiated animals maintained at lower altitudes. The large size of adipocytes required the use of log scales for both forward-and side-scatter data collection.

In samples costained with propidium iodide (PI), all $\mathrm{GFP}^{+}$particles exhibited bright PI fluorescence, indicating the presence of nuclei in the particles. This factor along with the large size (high forward scatter) indicates that the adipocytes not lysed by digestion/flotation remained intact during their passage through the sorter. However, visual examination of post-sort samples revealed 


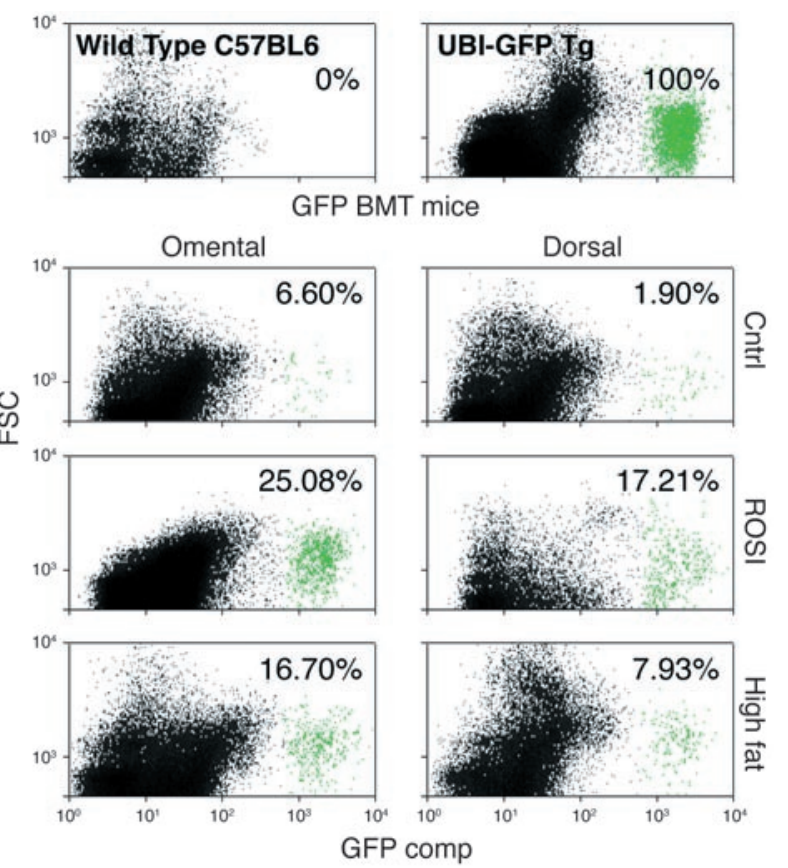

the presence of a substantial number (approximately 50\%-70\% of $\mathrm{GFP}^{+}$particles) of free nuclei. Thus, only $30 \%-50 \%$ of the adipocytes survived the sort intact. The remaining cells were probably disrupted by the high velocity with which they left the sorter and impacted the collection tube/solution.

Figure 3 shows the flow cytometric scattergram analysis on collagenase-liberated adipocytes from a nontransplanted, wild-type C57BL/ 6 mouse in which no GFP+ adipocytes were detected, compared with a sample from a GFP-expressing transgenic mouse in which all adipocytes expressed GFP. By comparison, samples from GFP+ BMT chimeric mice fed control diet revealed the presence of a small number ( $<10 \%$ of total cells) of GFP+ adipocytes in either omental or intrascapular fat. Samples from ROSI-treated animals had increased numbers of GFP+ adipocytes, ranging from approximately $15 \%$ to $30 \%$ of total cells. Samples from high-fat diet-fed animals demonstrated more GFP+ adipocytes than control samples but fewer than samples from ROSI-treated animals.

Duplicate samples of saponin-permeabilized isolated adipocytes were stained with PI and analyzed by flow cytometry for GFP and DNA content to assess the nuclear ploidy as an indicator of cell fusion events (19). Flow cytometric analysis with singlet discrimination demonstrated that $0.5 \%-1.5 \%$ of the $\mathrm{GFP}^{+}$adipocyte population displayed multinuclear content characteristic of increased nuclear ploidy or putative cell fusion events (Figure 4). This percentage of fused cells was too small to account for the large number of $\mathrm{GFP}^{+}$adipocytes detected by FACS analysis, indicating that the $\mathrm{GFP}^{+} \mathrm{ML}$ adipocytes arose from differentiation of BM-derived circulating precursors into ML adipocytes. These data also exclude the possibility that adipocytes become $\mathrm{GFP}^{+}$due to the adherence of $\mathrm{GFP}^{+}$circulating cells such as macrophages to ML adipocytes.

Preliminary phenotypic evaluation of $\mathrm{GFP}^{+} \mathrm{ML}$ adipocytes. Microscopic examination of isolated $\mathrm{GFP}^{+}$adipocytes confirmed their ML appearance, which is similar to that of brown fat cells but markedly different from that of unilocular white adipocytes (Figure $5 \mathrm{~A}$ ). Figure $5 \mathrm{~B}$ shows a phase-contrast image of a cluster of isolated adipocytes superimposed on a fluorescence image, dem-

\section{Figure 3}

FACS analysis of GFP+ adipocytes. Adipocytes were isolated from a nontransgenic, nontransplanted (WT C57BL/6) mouse (as a negative control); a UBI-GFP/BL6 transgenic (UBI-GFP Tg) donor mouse (as a positive control); and untreated GFP+ BMT mice (Cntrl), ROSI-treated mice, or mice fed a high-fat diet for 7 weeks. Adipocytes were isolated by collagenase digestion and flotation from omental or dorsal intrascapular depots. Shown are representative scattergrams in which green dots indicate GFP+ ${ }^{+}$cells and black dots represent either non$\mathrm{GFP}^{+}$cells, free lipid droplets, or debris. The average percentage of particles that were GFP+ is indicated at the top right of each scattergram. The results demonstrate that ROSI increases the number of $\mathrm{GFP}^{+}$adipocytes in the tissue samples. High-fat diet also increases $\mathrm{GFP}^{+}$adipocyte numbers but to lesser extent than ROSI treatment. GFP comp, GFP compensation.

onstrating the substantial number of $\mathrm{GFP}^{+}$adipocytes among the entire adipocyte population in ROSI-treated animals after 7 weeks of ROSI treatment.

Semiquantitative RT-PCR analysis demonstrated that the GFP ML adipocytes expressed several factors associated with terminal adipogenic development at levels comparable to those in white and brown adipocytes including CCAAT/enhancer-binding protein $\alpha$ $(\mathrm{C} / \mathrm{EBP} \alpha), \mathrm{PPAR} \gamma$, adiponectin, perilipin, and fatty acid-binding protein (FABP) (Figure 6A). ML adipocytes expressed approximately 5 -fold more UCP-1 RNA than white adipocytes, but only about $5 \%$ as much as detected in brown adipocytes. Leptin RNA levels in ML adipocytes were comparable to those in white adipocytes, while levels in brown adipocytes were approximately 5 -fold lower. Levels of $\beta 3$-adrenergic receptor ( $\beta 3$-AR) RNA in ML adipocytes were intermediate between those measured in white and brown adipocytes. Immunohistochemical analysis confirmed the results of the RT-PCR analysis. GFP ${ }^{+}$ML adipocytes expressed factors associated with terminal adipogenic development in mature white and brown adipocytes including $\mathrm{C} / \mathrm{EBP} \alpha, \operatorname{PPAR} \gamma$, adiponectin,

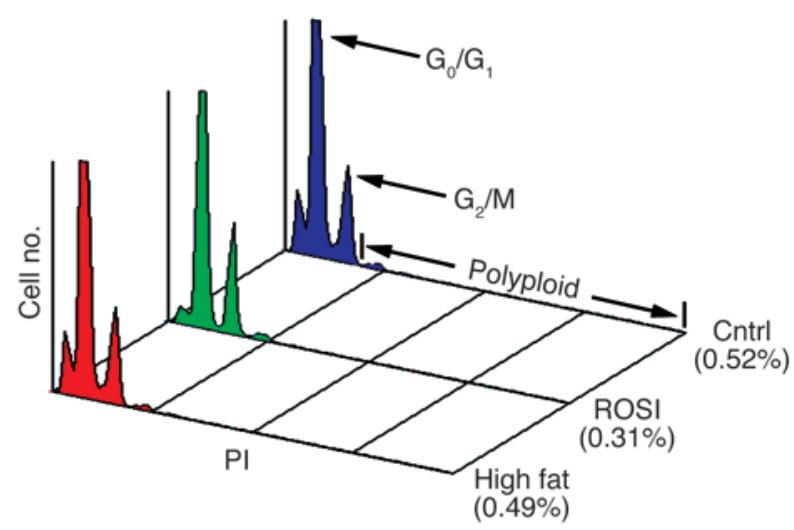

Figure 4

Nuclear DNA content analysis of GFP+ adipocytes. Adipocytes were isolated from $\mathrm{GFP}^{+} \mathrm{BMT}$ mice fed control, ROSI-impregnated, or highfat diets for 7 weeks. Adipocytes were permeablized with saponin and stained with PI. Flow cytometry was conducted with singlet discrimination. GFP-negative cells were excluded from the analysis. The position of polyploid (fused or multinuclear) cells or cells in the $G_{0} / G_{1}$ and $G_{2} / M$ regions of the cell cycle histogram are indicated. The small peak to the left of the $G_{0} / G_{1}$ peak in each histogram indicates apoptotic cells. The average percentage of polyploid cells in each treatment is indicated in parentheses below the label for each treatment histogram. 
A

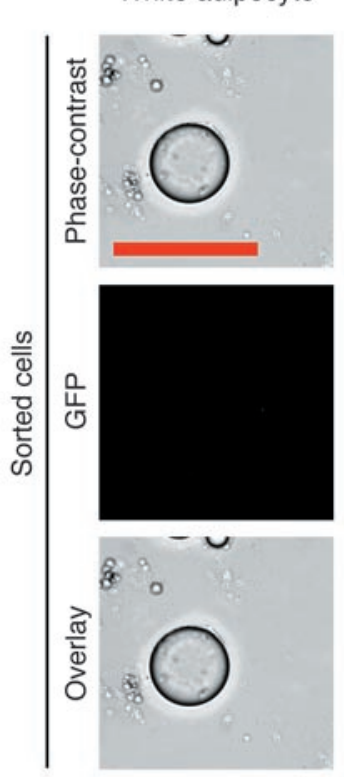

Brown adipocyte
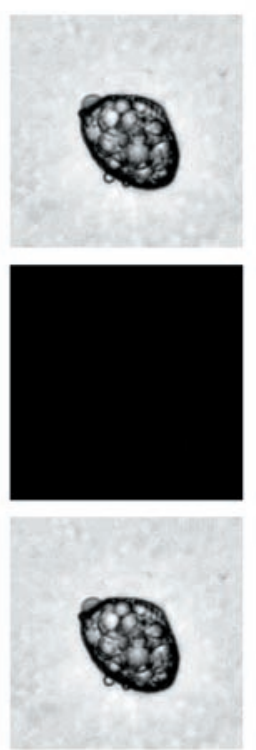
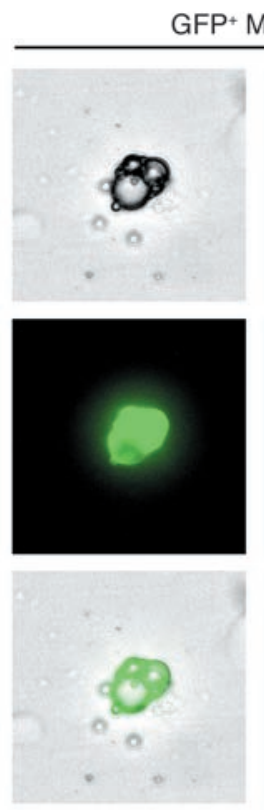

GFP + MLAs

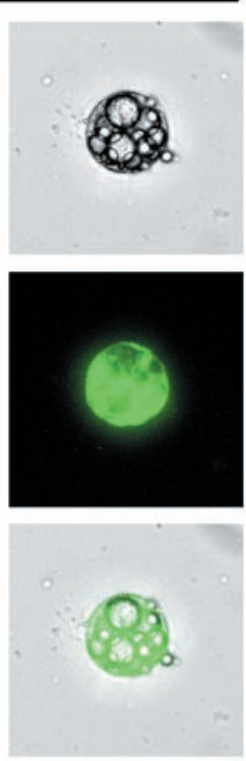

B Adipocytes
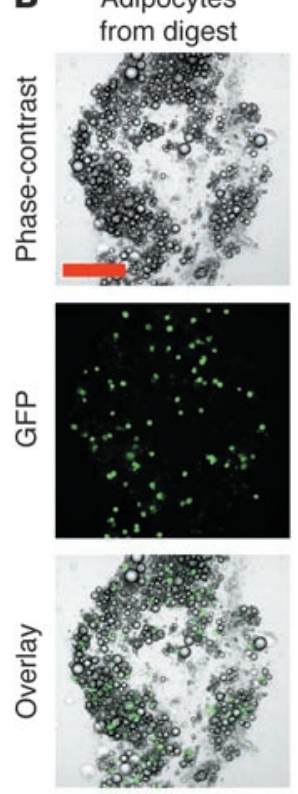

Figure 5

Microscopic observation of GFP+ML adipocytes isolated by collagenase digestion. (A) Omental and dorsal intrascapular adipose tissue was isolated from GFP+ BMT mice fed ROSI-impregnated chow for 7 weeks. The tissue was digested with collagenase, and adipocytes were isolated by flotation. Adipocytes were then subjected to flow sorting to separate GFP+ and GFP- cells. Isolated cells were examined by phase-contrast and fluorescence digital deconvolution microscopy to evaluate morphology and GFP expression. Shown are representative phase-contrast and fluorescence images of GFP+ ML adipocytes (MLAs) compared with a GFP- unilocular white adipocyte (from omental tissue) and a GFP- ML brown adipocyte (from dorsal intrascapular brown fat). Digital overlay of GFP fluorescence signal and phase-contrast images in shown. Scale bar (red): $100 \mu \mathrm{m}$. (B) Phase-contrast, fluorescence, and digital overlay images of adipocytes isolated by collagenase digestion and flotation from GFP+ BMT mice fed ROSI-impregnated diet for 7 weeks. The image shows the substantial number of GFP+ adipocytes present in the total adipocyte population.

and FABP (Supplemental Figure 2A). Western blot analysis of lysates from FACS-isolated $\mathrm{GFP}^{+} \mathrm{ML}$ adipocytes also detected expression of perilipin, leptin, and $\beta 3$-AR (Supplemental Figure $2 \mathrm{~B}$ ). Fluorescence deconvolution of omental white adipose tissue from mice treated with ROSI for 7 weeks and stained with Mitotracker dye showed that ML adipocytes had higher mitochondrial content than adjacent white adipocytes (Figure 6B).

Finally, Weisberg et al. (20) have reported increased numbers of macrophages in adipose tissue of obese individuals, and Cinti et al. (21) have demonstrated that macrophages can accumulate lipid from dying adipocytes. To determine whether $\mathrm{GFP}^{+} \mathrm{ML}$ adipocytes arise from macrophages, isolated $\mathrm{GFP}^{+}$adipocytes, stromal/vascular cells, or PBMCs were stained with antibodies to the panleukocyte marker CD45 and the macrophage marker CD11b and subjected to FACS analysis. Figure 7 shows that PBMCs contained a considerable number of $\mathrm{GFP}^{+}$cells expressing CD45 and/or CD11b. Likewise, the nonbuoyant stromal/vascular cell population contained $\mathrm{GFP}^{+}$cells with $\mathrm{CD} 45$ and/or CD11b surface markers. However, CD45 was undetectable in the $\mathrm{GFP}^{+} \mathrm{ML}$ adipocyte population with or without CD11b. A small percentage $(0.05 \%)$ of ML adipocytes expressed low levels of CD11b. These data suggest that ML adipocytes do not arise from macrophages.

\section{Discussion}

These studies demonstrate the development of ML adipocytes in both white and brown adipose tissue depots from BM-derived precursor cells and represent the first evidence to our knowledge that a unique population of fat cells arise from nonresident mesenchymal or hematopoietic progenitor cells. This observation is significant because although mesenchymal progenitors from $\mathrm{BM}$ and other tissues have been shown to differentiate into lipidladen cells with adipocyte characteristics (12), they have not been shown to mobilize from the $\mathrm{BM}$, take up residence in fat tissue, or become new fat cells in animal models. These data suggest that new fat cells arise not only from resident precursor cells, but also from other sources, such as BM-derived circulating progenitor cells. These data underscore the complexity of adipose tissue development and indicate that a broader understanding of BM-derived circulating progenitor cell mobilization, homing, and differentiation will be required to fully appreciate both normal adipose tissue development and the changes in adiposity that accompany weight gain, obesity, and lipodystrophic disorders.

Our preliminary phenotypic analysis shows that ML adipocytes express a number of adipocyte-related genes, including PPAR $\gamma$, $\mathrm{C} / \mathrm{EBP} \alpha$, adiponectin, perilipin, FABP, leptin, and $\beta 3$-AR. Although ML adipocytes express higher levels of UCP-1 that white adipocytes, the levels are much lower than those measured in brown adipocytes. Likewise, ML adipocyte RNA levels for leptin are comparable to those in white adipocytes, which are substantially higher than those in brown adipocytes. $\beta 3$-AR expression in $\mathrm{ML}$ adipocytes is intermediate between the levels measured in white and brown adipocytes. Thus, the general pattern of adipocyte gene expression in ML adipocytes is closer to that of white than of brown adipocytes. However, the high mitochondrial content of ML adipocytes is closer to that of brown adipocytes. Clearly, a more detailed phenotype analysis covering both gene expression 


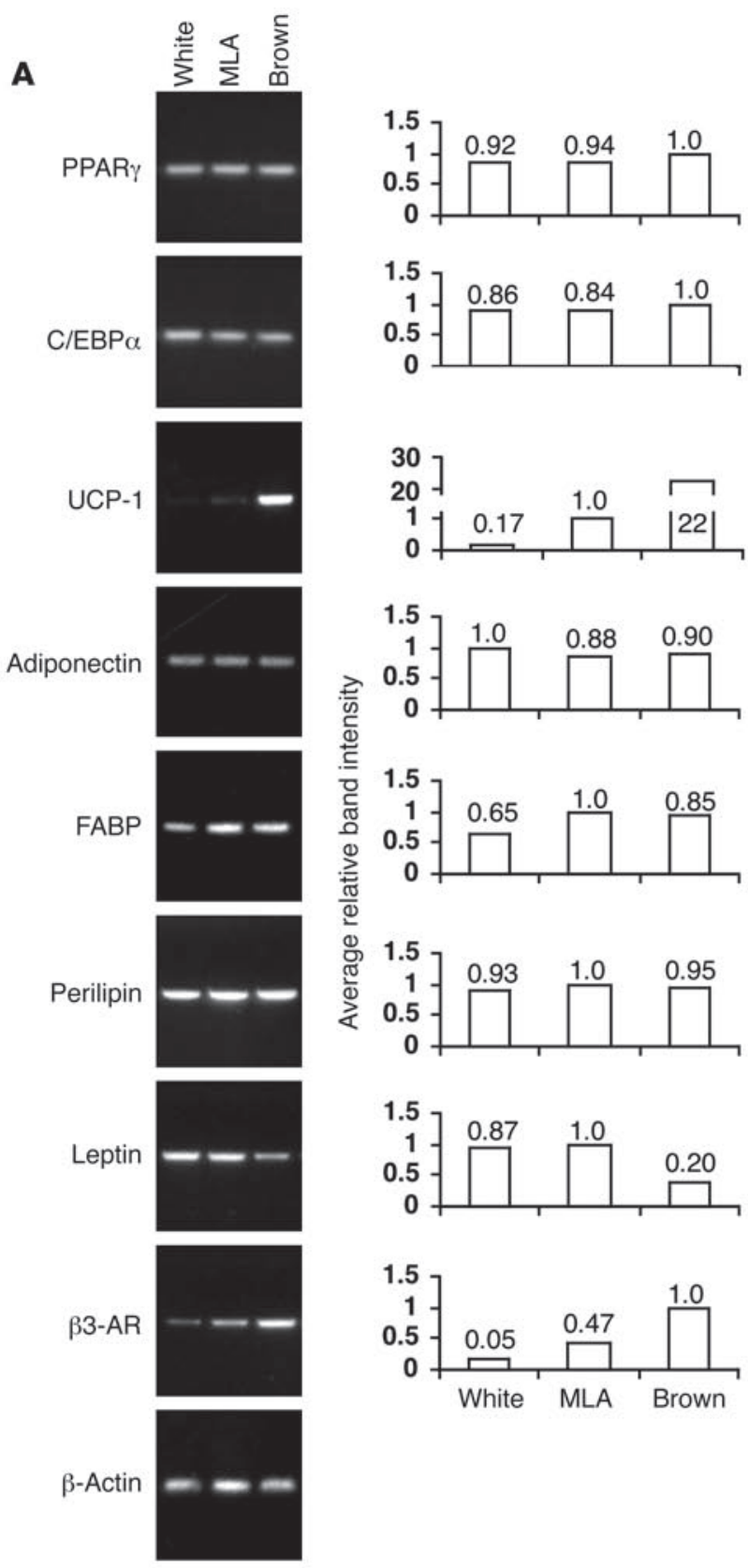

B
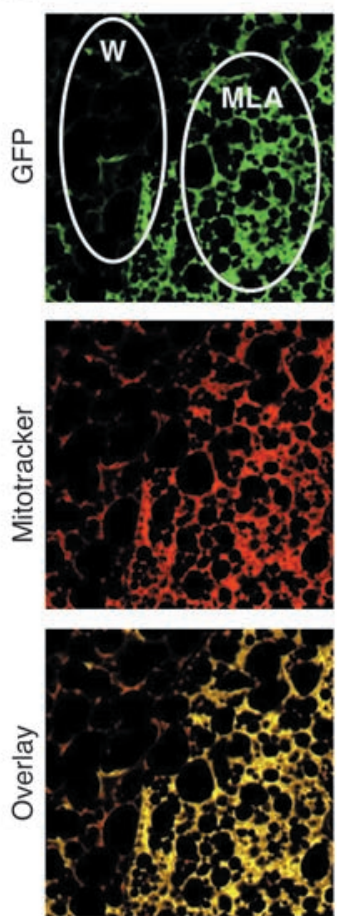

\section{Figure 6}

$\mathrm{GFP}^{+} \mathrm{ML}$ adipocytes express C/EBP $\alpha$, PPAR $\gamma$, adiponectin, FABP, perilipin, leptin, and $\beta 3-A R$ but not UCP-1 and have high mitochondrial content. (A) CDNA was prepared from RNA from white (from omental adipose tissue), brown (from dorsal intrascapular adipose tissue), and ML adipocytes isolated by collagenase digestion and flotation as described in Methods. Equal amounts of cDNA $(1 \mu \mathrm{g})$ were subjected to PCR with validated primer sets for the targets indicated to the left of each gel photograph. PCR reactions were then resolved on $2 \%$ agarose gels run in the presence of ethidium bromide. Fluorescence photographs of the gels were captured to computer, and band intensities were measured using ImageJ software. Representative gel photographs are shown in the left column. Densitomentry data were averaged over 3 experiments and corrected for differences in $\beta$-actin levels. Average band intensities are shown in the corresponding bar graphs to the right of each gel photograph. (B) Mitotracker Red 580 staining was performed on minced white adipose tissue fragments from GFP+ BMT mice fed ROSI-impregnated chow for 7 weeks. Representative fluorescence deconvolution images for GFP and Mitotracker signals, as well as a digital overlay of GFP and Mitotracker signals, are shown (yellow: GFP plus Mitotracker; red or orange: Mitotracker plus little or no GFP). The general location of white $(\mathrm{W})$ and $\mathrm{ML}$ adipocytes is indicated by the white ovals.

and metabolic activity is warranted in future studies. It will also be interesting to evaluate the contribution of BM-derived ML adipocytes to adipose tissue function. In this regard we have initiated studies to determine whether ML adipocytes can produce sufficient amounts of leptin to affect the adiposity, metabolism, and behavior of leptin-deficient mice.

The ML adipocytes observed in our studies are similar to ML white adipocytes (MLWAs) described by other laboratories. These investigators have reported the formation of large numbers of MLWAs in the adipose tissue of animals treated with $\beta 3$-AR agonists (22) or exposed to low-temperature environments (23). The MLWAs described by these investigators have multiple fat droplets, express certain adipocyte markers, and have high mitochondrial content but lack UCP-1. Thus, these cells have many of the char- acteristics of BM-derived ML adipocytes observed in our experiments. One major difference, however, is that MLWAs appear within several days of the initiation of $\beta 3$-AR agonist treatment or low-temperature exposure, while BM-derived ML adipocytes gradually appear over a period of several weeks with chronic TZD treatment or high-fat diet. MLWAs also constitute a very large proportion (perhaps 30\%) of adipose tissue from $\beta 3$-AR agonisttreated animals without apparent loss of white adipocytes due to apoptosis. Thus, MLWAs are believed to arise from the conversion of conventional white adipocytes to MLWAs, rather than arising from circulating or resident precursors.

Granneman et al. (24) have proposed that the MLWAs that develop in $\beta 3$-AR agonist-treated rodents may play a role in increasing insulin sensitivity by virtue of their elevated mito- 

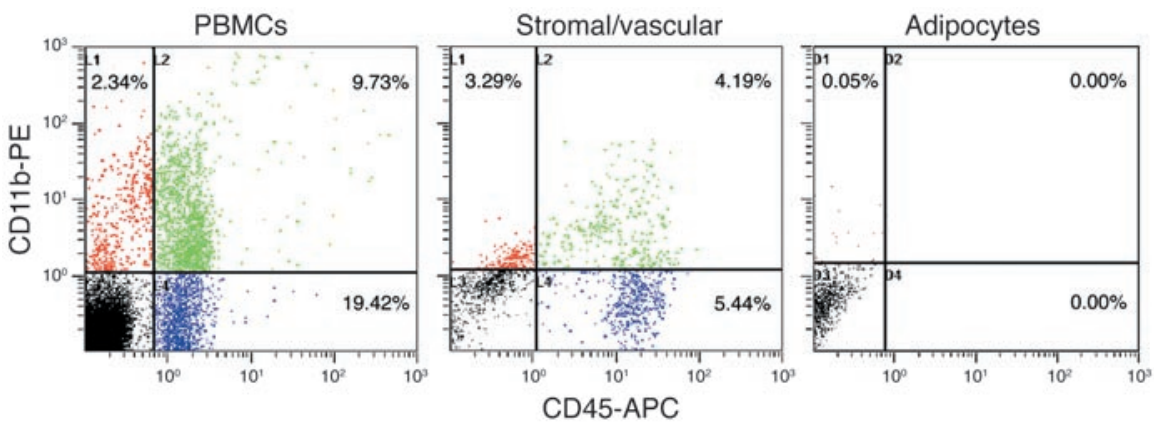

Figure 7

GFP+ adipocytes do not express CD45 or CD11b. PBMCs and omental adipose tissue were isolated from GFP+ BMT mice fed ROSI-impregnated chow for 7 weeks. The adipose tissue was digested with collagenase and nonbuoyant stromal/vascular cells, and buoyant adipocytes were separated by differential centrifugation. All 3 cell fractions were stained with APC-conjugated anti-CD45.2 (clone 104-2) antibodies and PE-conjugated anti-CD11b (clone M1/70) antibodies. The stained cell suspensions were subjected to FACS analysis for GFP+ excluded) expressing CD45 and/or CD11b. Representative scattergrams are shown, and the average percentage (from 3 independent sorts) of cells is indicated in the top left (CD11 $\mathrm{b}^{+}$), top right $\left(C D 45^{+}\right.$and $\left.C D 11 b^{+}\right)$, and bottom right $\left(C D 45^{+}\right)$quadrants. Gates were set and data were corrected using unstained cell suspensions or suspensions incubated with APC- and PE-conjugated isotype-matched control antibodies.

chondrial content, which might allow for enhanced clearance of circulating free fatty acids. This idea is supported by our observations showing that BM-derived ML adipocytes also have elevated mitochondrial content and that the appearance of these cells is promoted by the TZD ROSI, an insulin-sensitizing agent. Thus, in addition to improving insulin sensitivity in diabetic individuals by increasing adipogenesis and regulating cytokine expression, TZDs may also attenuate insulin resistance by lowering circulating free fatty acid levels via the mobilization of BM-derived cells to fat tissue, where they become ML adipocytes with a high fatty acid oxidation capacity. Why then is the appearance of ML adipocytes also elevated with high-fat feeding, a situation related to increased circulating levels of free fatty acids and insulin resistance? This phenomenon may reflect the ability of certain dietary fatty acids to serve as proadipogenic agents by acting as ligands for PPAR $\gamma$. The formation of ML adipocytes in this situation may then represent a protective mechanism to prevent an uncontrolled rise in the circulating free fatty acid levels with elevated dietary fat intake.

$\mathrm{GFP}^{+}$unilocular adipocytes were not observed in adipose tissue sections or detected by FACS analysis of isolated adipocytes, suggesting that unilocular adipocytes do not arise from nonresident progenitor cells. GFP- unilocular adipocytes could be recovered from the MoFlo cell sorter, indicating that the absence of $\mathrm{GFP}^{+}$unilocular adipocytes is not an artifact of the isolation process. Subsequent studies showed that $\mathrm{GFP}^{+}$unilocular cells were not present in BM-transplanted mice treated with ROSI or high-fat diet for 4-6 months, although $\mathrm{GFP}^{+} \mathrm{ML}$ adipocytes were present (data not shown). These results confirm that circulating progenitor cells do not differentiate into unilocular white adipocytes, nor do ML adipocytes that arise from BM-derived progenitor cells transform into unilocular cells with extended treatment. We have also looked for $\mathrm{GFP}^{+}$adipocytes in other tissues, including heart, skeletal muscle, kidney, and liver. No $\mathrm{GFP}^{+}$adipocytes have been detected in any of these tissues. This suggests that the mobilization of ML adipocyte progenitor cells is specific for adipose tissue.
Our data point to BM-derived circulating mesenchymal progenitor cells $\left(\mathrm{CD}^{-} 5^{-} \mathrm{Sca}-1^{+}\right)$as the source of $\mathrm{GFP}^{+} \mathrm{ML}$ adipocytes. Mesenchymal progenitor cells and $\mathrm{CD} 45^{-} \mathrm{Sca}-1^{+}$stem cells from BM and other tissues have been shown to differentiate into fat cells in vitro (13), making them potential precursors for the $\mathrm{GFP}^{+} \mathrm{ML}$ adipocytes. Also, the percentage of these cells in omental adipose tissue declined with ROSI treatment, consistent with a model in which these cells lose CD45 and Sca-1 expression as they take on adipocyte characteristics, while retaining their $\mathrm{GFP}^{+}$ expression. Interestingly, the mobilization and trafficking of mesenchymal progenitor cells in the circulation have not been previously reported, and their increased presence in the peripheral circulation of ROSI-treated animals is, to our knowledge, a previously unobserved phenomenon.

We also noted an increase in the number of circulating cells with markers characteristic of hematopoietic progenitor cells $\left(\mathrm{CD} 45^{+} \mathrm{c}-\mathrm{kit}^{+}\right)$at the 3 -week time point. CD $45^{+} \mathrm{c}-\mathrm{kit}{ }^{+}$cells are known to traffic from the BM and enter the peripheral circulation and can differentiate into cells with endothelial (25), smooth muscle (26), and neuronal (27) phenotypes. However, to our knowledge, these cells have not been shown to acquire adipocyte characteristics. We are currently performing BM transplants with mixtures of $\mathrm{GFP}^{+} \mathrm{CD}^{+} 5^{+}$(hematopoietic) and $\mathrm{GFP}^{-} \mathrm{CD} 45^{-}$(mesenchymal) cells, or alternately, $\mathrm{GFP}^{-} \mathrm{CD} 45^{+}$and $\mathrm{GFP}^{+} \mathrm{CD} 45^{-}$cells to determine whether hematopoietic or mesenchymal precursors give rise to $\mathrm{GFP}^{+} \mathrm{ML}$ adipocytes. These studies will be the first step in identifying the ML adipocyte progenitor cell population.

Finally, it is worth noting that other nonresident cells could serve as a source of new adipocytes in adipose tissue. Recently, Hong et al. (28) reported that circulating human $\mathrm{CD} 45^{+}$fibrocytes were capable of differentiating into adipocytes in culture. These adipocytes could form adipose tissue composed primarily of unilocular adipocytes when injected subcutaneously into nude mice. However, the ability of these fibrocytes to traffic to adipose tissue and differentiate into adipocytes in the rodent model was not explored. Another possibility is that new adipocytes could arise from cells of the promyelocytic lineage. Weisberg et al. (20) have reported an increase in the number of macrophages in adipose tissue with high-fat feeding. Cinti et al. (21) have demonstrated that these macrophages are frequently associated with necrotic adipocytes, from which they acquire triglycerides and form lipid droplets. However, these macrophages remain relatively small and nonbuoyant, are largely multinucleate, and do not express certain adipocyte markers such as perilipin (20). Therefore, it is highly unlikely that ML adipocytes arise from promyelocytic progenitor cells. This contention is confirmed by our data showing the absence of macrophage and leukocyte markers on $\mathrm{GFP}^{+} \mathrm{ML}$ adipocytes.

In conclusion, our experiments demonstrate the appearance of ML adipocytes from BM-derived progenitor cells. This phenomenon is stimulated by ROSI treatment and to a lesser extent by high-fat feeding. The appearance of these cells correlates with increased numbers of circulating mesenchymal and hematopoietic 
progenitor cells that may be the precursors for the ML adipocytes. The ML adipocytes express conventional adipocyte markers, have high levels of mitochondria, but lack expression of UCP-1.

\section{Methods}

Materials. PE-conjugated anti-mouse antibodies against Sca-1, c-kit, CD34, CD11b (clone M1/70), CD14, Gr-1, Thy-1, and B220; allophycocyaninconjugated (APC-conjugated) anti-mouse CD45 antibody; and APC- and PE-labeled isotype-matched control antibodies were purchased from BD Biosciences. APC-conjugated monoclonal CD45.2 (clone 104-2) antibodies were purchased from Abcam. Rabbit polyclonal anti-GFP, anti-UCP-1, anti-perilipin A, and anti-CD11b antibodies were purchased from Abcam. Polyclonal antibodies against leptin, C/EBP $\alpha$, and PPAR $\gamma$ were obtained from Santa Cruz Biotechnology Inc. Polyclonal antibodies against adiponectin were purchased from Sigma-Aldrich, antibodies against $\beta 3$-AR were purchased from Alpha Diagnostic International, and antibodies against FABP were purchased from Cayman Chemical. Mitotracker Red 580- and Alexa Fluor 594- and Alexa Fluor 488-conjugated secondary antibodies were from Invitrogen. Validated PCR primer sets for PPAR $\gamma$, $\mathrm{C} / \mathrm{EBP} \alpha, \mathrm{UCP}-1$, adiponectin, FABP, perilipin, leptin, $\beta 3$-AR, and $\beta$-actin were obtained from SuperArray Bioscience Corp. Diet 5008 (6.5\% fat, $3.31 \mathrm{kcal} / \mathrm{g}$ ) impregnated with $0.015 \%$ ROSI (Avandia; GlaxoSmithKline) was prepared by TestDiet, and high-fat diet TD 01640 (20\% fat, $4.3 \mathrm{kcal} / \mathrm{g})$ was from Harlan Teklad.

GFP BM transplantation, animal treatments, and tissue recovery. Six- to 8-weekold female C57BL/6 and C57BL/6-Tg(UBC-GFP)30Scha/J (UBI-GFP/BL6) mice were purchased from The Jackson Laboratory and housed in the Center for Laboratory Animal Care facility at the University of Colorado Health Sciences Center. All procedures and treatments were performed under a protocol approved by the University of Colorado Institutional Animal Care and Use Committee. Recipient mice were irradiated with a split dose of $6 \mathrm{~Gy}$ using a cesium source. Immediately following irradiation, mice were injected in the retroorbital venous plexus with $5 \times 10^{6} \mathrm{BM}$ cells from homozygous female UBI-GFP/BL6 mice. At 8 weeks after transplantation, mice were assessed for chimerism by FACS analysis of peripheral blood. As shown in Supplemental Figure 1A, in the transplanted mice, more than 95\% of peripheral blood cells expressed GFP.

Transplanted mice were maintained in a climate-controlled room with alternating 12-hour periods of light and dark. The mice were fed control, ROSIimpregnated (approximate dose $15 \mathrm{mg} / \mathrm{kg} / \mathrm{d}$ ), or high-fat diet ad libitum.

At various time points, mice were anesthetized by pentobarbital overdose and euthanized by exsanguination. Blood was collected by infusing a solution of PBS containing 5 mM EDTA into the left ventricle while simultaneously collecting blood from the right ventricle. Omental, perinephric, and dorsal intrascapular fat pads were excised, and portions were fixed in $4 \%$ paraformaldehyde in PBS overnight and then embedded in paraffin for sectioning. Remaining portions were subjected to collagenase digestion or flash frozen in liquid nitrogen.

Flow cytometry and FACS. Adipocyte and stromal/vascular fractions from mouse adipose tissue were prepared by collagenase digestion. GFP- white adipocytes were recovered from omental white adipose tissue. GFP- brown adipocytes were isolated from dorsal intrascapular adipose tissue after all white adipose tissue was removed under a dissecting microscope. Briefly, 1g portions of tissue were suspended in HBSS containing $2 \mathrm{mg} / \mathrm{ml}$ collagenase type I (Invitrogen) and $4 \mathrm{mg} / \mathrm{ml} \mathrm{BSA}$ and agitated for 1 hour at $37^{\circ} \mathrm{C}$. The suspension was filtered through a $200-\mu \mathrm{m}$ sieve and allowed to stand for 5 minutes to allow the buoyant adipocytes to rise to the top of the solution. Suspensions were then centrifuged at $500 \mathrm{~g}$ for 5 minutes to pellet the stromal/vascular cells. The adipocyte layer was gently recovered, and the remaining supernatant was removed by aspiration. Both the adipocyte layer and pelleted stromal cells were separately resuspended in HBSS/BSA. The stromal cells were recovered by centrifugation and the adipocytes by flotation, and both were resuspended in HBSS/BSA at $2 \times 10^{6}$ cells $/ \mathrm{ml}$. PBMCs were isolated from peripheral blood by differential centrifugation with Percoll (Amersham Biosciences). PBMCs were resuspended in PBS containing $5 \%$ FBS at $2 \times 10^{6}$ cells $/ \mathrm{ml}$.

For flow cytometry, PBMCs or stromal/vascular cells were stained with antibodies against the cell-surface markers indicated in the table and figure legends. Cell suspensions $(0.5 \mathrm{ml})$ were added to microcentrifuge tubes containing $1 \mu \mathrm{g}$ of each APC- or PE-conjugated antibody and incubated at $4^{\circ} \mathrm{C}$ for 20 minutes. Cells were then pelleted at $500 \mathrm{~g}$, resuspended in cold $\mathrm{PBS} / 5 \% \mathrm{FBS}$, and held at $4^{\circ} \mathrm{C}$ for same-day analysis. Controls for FACS analysis included unstained cells and cell suspensions incubated with APCor PE-conjugated isotype-matched control antibodies. Cells were analyzed on a Beckman Coulter FC 500 flow cytometer.

Ploidy analysis was performed on adipocytes suspended in PBS containing saponin ( $0.3 \%$ final concentration), PI $(25 \mu \mathrm{g} / \mathrm{ml})$, and EDTA $(0.1 \mathrm{mM})$. Cells were analyzed by flow cytometry with singlet discrimination to detect $\mathrm{GFP}^{+}$and PI-stained, fused, or multinuclear cells.

Adipocytes were sorted using a MoFlo cell sorter with Summit 4.0 software (Dako). The MoFlo was equipped with a Spectra-Physics air-cooled argon laser operating at $43 \mathrm{~mW}$ at $488 \mathrm{~nm}$. A $100-\mu \mathrm{m}$ nozzle tip was used with a sheath pressure of $30 \mathrm{psi}$ and a drop drive frequency of $46,700 \mathrm{~Hz}$ and amplitude of $15 \mathrm{~V}$. The sheath fluid consisted of IsoFlow (Beckman Coulter). The sample and collection tubes were maintained at $5^{\circ} \mathrm{C}$ using an attached Haake recirculating water bath. To keep cells in suspension, the MoFlo was equipped with a SmartSampler (Dako) sample station with the sample agitation set to maintain an agitation cycle of 4 seconds on and 5 seconds off. The sample flow rate was set to a pressure differential of less than 0.4 psi. $\mathrm{GFP}^{+}$cells were collected using a 530/40 bandpass filter with a voltage of 550 . Sort mode was set to Purify 1 . Forward-angle light scatter and side light scatter data were collected using log scales. Appropriate signal compensation was set using single-color control samples. For PCR analysis of adipocyte markers, cells were collected in tubes containing RNAlater (Ambion). Cells were collected in RIPA buffer for Western blot studies. Finally, for morphological studies, cells were collected in PBS containing 10\% FCS.

$R T-P C R$. cDNA was prepared from collagen/flotation-isolated white, brown, or ML adipocytes using Cells-to-cDNA II (Ambion) reagents according to the manufacturer's instructions. PCR amplification was performed with $3 \mu \mathrm{l}$ transcribed cDNA and 1 pmol of each primer for 30 cycles: hotstart at $94^{\circ} \mathrm{C}$ for 1 minute, denaturation at $95^{\circ} \mathrm{C}$ for 1 minute, annealing at $55^{\circ} \mathrm{C}$ for 30 seconds, and elongation at $72^{\circ} \mathrm{C}$ for 2 minutes. Negative PCR controls included omission of reverse transcriptase or omission of cDNA. $\beta$-Actin primers were used to validate each batch of template before use. PCR products were resolved on $2 \%$ agarose gels containing ethidium bromide and photographed under ultraviolet illumination. Photographs of gels were captured to a Macintosh PowerBook G4 laptop computer (Apple Computer Inc.), and densitometry of the PCR product bands was performed with NIH ImageJ software (http://rsb.info.nih.gov/ij/).

Immunohistochemistry and microscopy. Five-micrometer sections of paraformaldehyde-fixed, paraffin-embedded adipose tissue were deparaffinized with Hemo-D (Scientific Safety Solvents) and rehydrated in a graded ethanol/water series. Sections were subjected to antigen retrieval in citrate buffer in a microwaveable pressure cooker for 20 minutes. Sections were blocked with PBS containing $5 \%$ horse serum for 30 minutes at room temperature. The sections were incubated overnight in PBS/5\% FBS at $4{ }^{\circ} \mathrm{C}$ with the primary antibodies indicated in the figure legends. The sections were then washed and incubated with the indicated Alexa Fluoror HRP-conjugated secondary antibodies for 1 hour at room temperature. 
HRP was detected with NBT reagents from Vector Laboratories. Mitotracker Red 580 staining was performed on minced portions of unfixed adipose tissue according to the supplier's recommendations.

Microscopy was performed on a Nikon TE2000-U inverted epifluorescent microscope with remote focus device. Phase-contrast and fluorescent images were captured to a personal computer with a SPOT RT/KE monochrome camera (Diagnostic Instruments). Images were analyzed and processed with MetaMorph 6.1 software (Molecular Devices).

Western blot analysis. Stromal/vascular cells, GFP- white and brown adipocytes, and $\mathrm{GFP}^{+} \mathrm{ML}$ adipocytes were prepared by collagenase digestion/flotation and FACS sorting as described above. Equal numbers of cells were lysed in PBS containing 1\% Triton X-100 by Dounce homogenization. Lysates were clarified by centrifugation at 13,000 $\mathrm{g}$ for $5 \mathrm{~min}$ utes. Equal amounts of supernatant proteins were mixed with Laemmli SDS loading buffer, resolved on 10\% SDS-polyacrylamide gels, and transferred to PVDF membranes. Equal protein loading and efficiency of transfer to PVDF membranes was verified by brief staining of the blots with Ponceau red stain. The blots were blocked with PBS containing $5 \%$ dry milk and $0.1 \%$ Tween- 20 and then treated with antibodies that recognize the target proteins indicated in each figure overnight at

$4^{\circ} \mathrm{C}$. The blots were washed and subsequently treated with appropriate secondary antibodies conjugated to HRP. After the blots were washed, specific immune complexes were visualized with SuperSignal West Pico Chemiluminescent Substrate (Pierce Biotechnology).

Statistics. Data averaged from at least 3 samples for each treatment condition were compared with averaged data from untreated controls using the 2-tailed Student's $t$ test. Differences were considered statistically significant at $P \leq 0.05$.

\section{Acknowledgments}

This work was funded by NIH grant DK53969 (to D.J. Klemm).

Received for publication March 14, 2006, and accepted in revised form September 12, 2006.

Address correspondence to: Dwight J. Klemm, Cardiovascular Pulmonary Research, University of Colorado Health Sciences Center, 4200 East Ninth Avenue, Campus Box B-133, Denver, Colorado 80262, USA. Phone: (303) 315-8107; Fax: (303) 315-4871; E-mail: Dwight.Klemm@UCHSC.edu.

1. Steppan, C.M., and Lazar, M.A. 2002. Resistin and obesity-associated insulin resistance. Trends Endocrinol. Metab. 13:18-23.

2. Poissonnet, C.M., Burdi, A.R., and Garn, S.M. 1984. The chronology of adipose tissue appearance and distribution in human fetus. Early Hum. Dev. 10:1-11.

3. Poissonnet, C.M., Lavelle, M., and Burdi, A.R. 1988. Growth and development of adipose tissue.J. Pediatr. 113:1-9.

4. Ailhaud, G., Grimaldi, P., and Negrel, R. 1992. Cellular and molecular aspects of adipose tissue development. Annu. Rev. Nutr. 12:207-233.

5. Garaulet, M., Hernandez-Morante, J.J., Lujan, J., Tebar, F.J., and Zamora, S. 2006. Relationship between fat cell size and number and fatty acid composition in adipose tissue from different fat depots in overweight/obese humans. Int. J. Obes. (Lond.). 30:899-905.

6. Marques, B.G., Hausman, D.B., and Martin, R.J. 1998. Association of fat cell size and paracrine growth factors in development of hyperplastic obesity. Am. J. Physiol. 275:R1891-R1908.

7. Fonseca, V. 2003. Effect of thiazolidinediones on body weight in patients with diabetes mellitus. Am. J. Med. 115(Suppl. 8A):42S-48S.

8. Lehmann, J.M., et al. 1995. An antidiabetic thiazolidinedione is a high affinity ligand for peroxisome-proliferator-activated-receptor $\gamma$.J. Biol. Chem. 270:12953-12956.

9. Kletzien, R.F., Clarke, S.D., and Ulrich, R.G. 1992. Enhancement of adipocyte differentiation by an insulin-sensitizing agent. Mol. Pharmacol. 41:393-398.

10. Sandouk, T., Reda, D., and Hofman, C. 1993. Antidiabetic agent pioglitazone enhances adipocyte differentiation of 3T3-F442A cells. Am. J. Physiol.
264:C1600-C1608.

11. Bosnakovski, D., et al. 2005. Isolation and multilineage differentiation of bovine bone marrow mesenchymal stem cells. Cell Tissue Res. 319:243-253.

12. Dicker, A., et al. 2005. Functional studies of mesenchymal stem cells derived from adult human adipose tissue. Exp. Cell Res. 308:283-290.

13. Peister, A., et al. 2004. Adult stem cells from bone marrow (MSCs) isolated from different strains of inbred mice vary in surface epitopes, rates of proliferation, and differentiation potential. Blood. 103:1662-1668.

14. Tanaka, T., et al. 2005. Therapeutic potential of thiazolidinediones in activation of peroxisome proliferator-activated receptor gamma for monocyte recruitment and endothelial regeneration. Eur. J. Pharmacol. 508:255-265.

15. Gosset, P., et al. 2001. Peroxisome proliferator-activated receptor gamma activators affect the maturation of human monocyte-derived dendritic cells. Eur. J. Immunol. 31:2857-2865

16. Pistrosch, F., et al. 2005. PPARgamma-agonist rosiglitazone increases number and migratory activity of cultured endothelial progenitor cells. Atherosclerosis. 183:163-167.

17. Sidhu, J.S., Cowan, D., Tooze, J.A., and Kaski, J.C. 2004. Peroxisome proliferator-activated receptorgamma agonist rosiglitazone reduces circulating platelet activity in patients without diabetes mellitus who have coronary artery disease. Am. Heart J. 147:e25.

18. Schaefer, B.C., Schaefer, M.L., Kappler, J.W., Marrack, P., and Kedl, R.M. 2001. Observation of antigen-dependent CD8+ T-cell/dendritic cell interactions in vivo. Cell. Immunol. 214:110-122.

19. Mayhew, C.N., et al. 2005. Liver-specific pRB loss results in ectopic cell cycle entry and aberrant ploidy.
Cancer Res. 65:4568-4577.

20. Weisberg, S.P., et al. 2003. Obesity is associated with macrophage accumulation in adipose tissue. J. Clin. Invest. 112:1796-1808. doi:10.1172/JCI200319246.

21. Cinti, S., et al. 2005. Adipocyte death defines macrophage localization and function in adipose tissue of obese mice and humans. J. Lipid Res. 46:2347-2355.

22. Granneman, J.G., Li, P., Lu, Y., and Tilak, J. 2004. Seeing the trees in the forest: selective electroporation of adipocytes within adipose tissue. Am. J. Physiol. Endocrinol. Metab. 287:E574-E582.

23. Loncar, D., Afzelius, B.A., and Cannon, B. 1988. Epididymal white adipose tissue after cold stress in rats. II. Mitochondrial changes. J. Ultrastruct. Mol. Struct. Res. 101:199-209.

24. Granneman, J.G., Li, P., Zhu, Z., and Lu, Y. 2005. Metabolic and cellular plasticity in white adipose tissue I: effects of beta3-adrenergic receptor activation. Am. J. Physiol. Endocrinol. Metab. 289:E608-E616.

25. Kaushal, S., et al. 2001. Functional small-diameter neovessels created using endothelial progenitor cells expanded ex vivo. Nat. Med. 7:1035-1040.

26. Sata, M., et al. 2002. Hematopoietic stem cells differentiate into vascular cells that participate in the pathogenesis of atherosclerosis. Nat. Med. 8:403-409.

27. Koshizuka, S., et al. 2004. Transplanted hematopoietic stem cells from bone marrow differentiate into neural lineage cells and promote functional recovery after spinal cord injury in mice. J. Neuropathol. Exp. Neurol. 63:64-72.

28. Hong, K.M., Buridick, M.D., Phillips, R.J., Heber, D., and Strieter, R.M. 2005. Characterization of human fibrocytes as circulating adipocyte progenitors and the formation of human adipose tissue in SCID mice. FASEB J. 19:2029-2031. 